\title{
BOUNDS FOR EIGENVALUES OF A GRAPH
}

\section{RAVINDER KUMAR}

Abstract. New lower bounds for eigenvalues of a simple graph are derived. Upper and lower bounds for eigenvalues of bipartite graphs are presented in terms of traces and degree of vertices. Finally a non-trivial lower bound for the algebraic connectivity of a connected graph is given.

Mathematics subject classification (2010): 05C50, 15A42, 15A36.

Keywords and phrases: eigenvalues, spread, laplacian, algebraic connectivity.

\section{REFERENCES}

[1] D. M. Cvetković, M. Doob And H. Sachs, Spectra of Graphs, Academic Press, 1979.

[2] D. CvetKović And P. Rowlinson, The largest eigenvalue of a graph: A Survey, Linear and Multilinear Algebra, 28 (1990), 3-33.

[3] C. Godsil And G. RoyLe, Algebraic Graph Theory, Springer-Verlag, New York, 2001.

[4] F. HARARY, Graph Theory, Addison-Wesley, 1969.

[5] Y. P. HONG AND C.-T. PAN, A lower bound for smallest singular value, Linear Algebra and Applications, 172 (1992), 27-32.

[6] L. Mirs Ky, Inequalities for normal and Hermitian matrices, Duke Math. J., 24 (1957), 591-599.

[7] C. R. Johnson, R. Kumar And H. Wolkowicz, Lower Bounds for the Spread of a matrix, Linear Algebra and Applications, 71 (1985), 161-173.

[8] D. B. West, Introduction to Graph Theory, Second Edition, Prentice-Hall of India Pvt. Ltd., 2003.

[9] H. Wolkowicz and G. P. H. StYan, Bounds for Eigenvalues Using Traces, Linear Algebra and Applications, 29 (1980), 471-506. 